VOLUME 29 (2021) 50-62

DOI: $10.24330 /$ ieja. 852003

\title{
CLASSIFICATION OF SOME SPECIAL GENERALIZED DERIVATIONS
}

\author{
M. A. Idrissi and L. Oukhtite \\ Received: 15 October 2019; Revised: 22 July 2020; Accepted: 27 July 2020 \\ Communicated by A. Çiğdem Özcan
}

\begin{abstract}
The purpose of the present paper is to classify generalized derivations satisfying more specific algebraic identities in a prime ring with involution of the second kind. Some well-known results characterizing commutativity of prime rings by derivations have been generalized by using generalized derivation.
\end{abstract}

Mathematics Subject Classification (2020): 16N60, 16W10, 16W25

Keywords: Prime ring, involution, commutativity, derivation, generalized derivation

\section{Introduction}

Throughout this paper, $R$ will represent an associative ring with center $Z(R)$. For any $x, y \in R$ the symbol $[x, y]$ will denote the commutator $x y-y x$; while the symbol $x \circ y$ will stand for the anti-commutator $x y+y x$. Recall that $R$ is prime if $a R b=(0)$ with $a, b \in R$ implies $a=0$ or $b=0$.

An additive map $*: R \longrightarrow R$ is called an involution if $*$ is an anti-automorphism of order 2. An element $x$ of a ring $R$ with involution $*$ is said to be hermitian if $x^{*}=x$ and skew-hermitian if $x^{*}=-x$. The sets of all hermitian and skew-hermitian elements of $R$ will be denoted by $H(R)$ and $S(R)$, respectively. The involution is said to be of the first kind if $Z(R) \subseteq H(R)$, otherwise it is said to be of the second kind. In the later case it is straightforward to check that $S(R) \cap Z(R) \neq(0)$.

An additive map $d: R \longrightarrow R$ is called a derivation if $d(x y)=d(x) y+x d(y)$ for all $x, y \in R$. Let $a \in R$ be a fixed element, the map $d: R \longrightarrow R$ defined by $d(x)=[a, x]$ for all $x \in R$ is a derivation on $R$, and it is called the inner derivation induced by $a$.

Many results in literature indicate how the global structure of a ring $R$ is often tightly connected to the behavior of additive mappings defined on $R$. A well known result due to Posner [14] states that if $d$ is a derivation of a prime ring $R$ such that $[d(x), x] \in Z(R)$ for all $x \in R$, then either $d=0$ or $R$ is commutative. In 
[9] Lanski generalizes the result of Posner by considering a derivation $d$ such that $[d(x), x] \in Z(R)$ for all $x$ in a nonzero Lie ideal $U$ of $R$.

More recently several authors consider similar situation in the case the derivation $d$ is replaced by a generalized derivation. More specifically an additive map $F: R \longrightarrow R$ is said to be a generalized derivation if there exists a derivation $d$ of $R$ such that $F(x y)=F(x) y+x d(y)$ for all $x, y \in R$. Basic examples of generalized derivations are the usual derivations on $R$ and left $R$-module mappings from $R$ into itself. An important example is a map of the form $F(x)=a x+x b$ for some elements $a, b \in R$; such generalized derivations are called inner. Generalized derivations have been primarily studied on operator algebras thus any investigation from the algebraic point of view might be interesting (see for example [6], [7] and [11]).

During the last two decades, many authors have studied commutativity of prime and semi-prime rings admitting suitably constrained additive mappings acting on appropriate subsets of the rings. Moreover, many of obtained results extend other ones previously proven just for the action of the considered mapping on the whole ring. In this direction, the recent literature contains numerous results on commutativity in prime and semi-prime rings admitting suitably constrained derivations and generalized derivations, and several authors have improved these results by considering rings with involution (for example, see [1], [2], [13]).

The present paper is motivated by the previous results and we here continue this line of investigation by considering new classes of generalized derivations.

\section{Main results}

We begin our discussion with the following known results which will be used extensively to prove our theorems.

Fact. Let $(R, *)$ be a 2-torsion free prime ring with involution of the second kind, then $Z(R) \cap H(R) \neq\{0\}$.

Proof. As $*$ is of the second kind, by definition, there exists a nonzero element $z$ in $Z(R)$ such that $z^{*} \neq z$. Setting $h=z z^{*}$, it is clear that $h \in Z(R) \cap H(R)$. Moreover, $h \neq 0$ otherwise $0=z z^{*}$ so that $z R z^{*}=\{0\}$ and the primeness of $R$ yields $z=0$, a contradiction. Hence $Z(R) \cap H(R) \neq\{0\}$.

Lemma 2.1. [12, Fact 1] Let $(R, *)$ be a 2-torsion free prime ring with involution and $d$ a derivation on $R$. Then $d(h)=0$ for all $h \in H(R) \cap Z(R)$ implies that $d(z)=0$ for all $z \in Z(R)$. 
Lemma 2.2. [4, Lemma] Let $R$ be a prime ring. If functions $F: R \longrightarrow R$ and $G: R \longrightarrow R$ are such that $F(x) y G(z)=G(x) y F(z)$ for all $x, y, z \in R$, and $F \neq 0$, then there exists $\lambda$ in the extended centroid of $R$ such that $F(x)=\lambda G(x)$ for all $x \in R$.

Lemma 2.3. Let $R$ be a 2-torsion free prime ring and $F: R \rightarrow R$ be a generalized derivation associated with a derivation $d$. Then the following assertions are equivalent:

(1) $F(x \circ y)=F(x) \circ y-d(y) \circ x$ for all $x, y \in R$.

(2) $F([x, y])=[F(x), y]-d(y) \circ x$ for all $x, y \in R$.

(3) $F([x, y])=[F(x), y]+[d(y), x]$ for all $x, y \in R$.

(4) There exists $\lambda$ in the extended centroid of $R$ such that $F(x)=\lambda x$ for all $x \in R$ (and therefore $d=0$ ).

Proof. It is enough to prove that each of (1), (2) and (3) implies (4). We first recall that the generalized derivation $F$ is of the form $F(x)=\lambda x+d(x)$ for all $x \in R$ and some $\lambda$ in the maximal left ring of quotients $Q(R)$ of $R$ by [11].

$(1) \Rightarrow(4)$ Using the above form of $F$ in the relation

$$
F(x \circ y)=F(x) \circ y-d(y) \circ x
$$

for all $x, y \in R$, one obtains

$$
([\lambda, y]+2 d(y)) x+x(2 d(y))=0 .
$$

Then by Lemma 4.5 in [5], we get

$$
[\lambda, y]+2 d(y)=-2 d(y) \in C
$$

for all $y \in R$. Hence we once see that $d=0$ and $\lambda \in C$, and so $F(x)=\lambda x$ for all $x \in R$.

$(2) \Rightarrow(4)$ Again, we get from the relation

$$
F([x, y])=[F(x), y]-d(y) \circ x
$$

for all $x, y \in R$ that

$$
[\lambda, y] x+x(-2 d(y))=0
$$

Then by Lemma 4.5 in [5], we get

$$
[\lambda, y]=2 d(y) \in C
$$


for all $y \in R$. Hence $d=0$, and then it follows that $\lambda \in C$ as we desired. $(3) \Rightarrow(4)$ Similar to above argument, the relation

$$
F([x, y])=[F(x), y]+[d(y), x]
$$

for all $x, y \in R$ leads us to

$$
([\lambda, y]+2 d(y)) x+x(-2 d(y))=0
$$

Another use of Lemma 4.5 in [5] results in

$$
[\lambda, y]+2 d(y)=2 d(y) \in C
$$

for all $y \in R$. Therefore $d=0$ and $\lambda \in C$.

In [3, Theorem 2.9] it is proved that if $R$ is a 2-torsion free semi-prime ring with a generalized derivation $F$ associated with a nonzero derivation $d$ such that $F(x \circ y)=F(x) \circ y-d(y) \circ x$ for all $x, y \in I$; where $I$ is a nonzero ideal of $R$, then $R$ contains a nonzero central ideal.

Motivated by [3], our purpose is to study the same identity on prime rings with involution. More precisely the following theorem classifies generalized derivations satisfying such condition.

Theorem 2.4. Let $(R, *)$ be a 2-torsion free prime ring with involution of the second kind. If $R$ admits a generalized derivation $F$ associated with a derivation d, then the following assertions are equivalent:

(1) $F\left(x \circ x^{*}\right)=F(x) \circ x^{*}-d\left(x^{*}\right) \circ x$ for all $x \in R$.

(2) $F\left(\left[x, x^{*}\right]\right)=\left[F(x), x^{*}\right]-d\left(x^{*}\right) \circ x$ for all $x \in R$.

(3) There exists $\lambda$ in the extended centroid of $R$ such that $F(x)=\lambda x$ for all $x \in R$.

Proof. We need only prove that (1) $\Rightarrow(3)$ and $(2) \Rightarrow(3)$.

$(1) \Rightarrow(3)$ We are given that

$$
F\left(x \circ x^{*}\right)=F(x) \circ x^{*}-d\left(x^{*}\right) \circ x \text { for all } x \in R .
$$

Linearizing the above relation we find that

$F\left(x \circ y^{*}\right)+F\left(y \circ x^{*}\right)=F(x) \circ y^{*}-d\left(x^{*}\right) \circ y+F(y) \circ x^{*}-d\left(y^{*}\right) \circ x$ for all $x, y \in R$, so that

$$
F(x \circ y)+F\left(y^{*} \circ x^{*}\right)=F(x) \circ y-d\left(x^{*}\right) \circ y^{*}+F\left(y^{*}\right) \circ x^{*}-d(y) \circ x
$$


Replacing $y$ by $y h$ in (2), where $h \in Z(R) \cap H(R) \backslash\{0\}$, one can obtain

$$
(x \circ y) d(h)=0 \text { for all } x, y \in R .
$$

Since $R$ is prime, then Eq. (3) assures that either $d(h)=0$ or $x \circ y=0$ which leads to $R=\{0\}$, a contradiction. Therefore we need consider that $d(h)=0$ for all $h \in Z(R) \cap H(R)$. Applying Lemma 2.1 one can see that $d(s)=0$ for all $s \in Z(R) \cap S(R)$.

Taking $y=y s$ in (2), where $s \in Z(R) \cap S(R) \backslash\{0\}$, we have

$$
F(x \circ y)-F\left(y^{*} \circ x^{*}\right)=F(x) \circ y+d\left(x^{*}\right) \circ y^{*}-F\left(y^{*}\right) \circ x^{*}-d(y) \circ x
$$

From EQs. (2) and (4) it follows that

$$
F(x \circ y)=F(x) \circ y-d(y) \circ x \text { for all } x, y \in R \text {. }
$$

By view of Lemma 2.3, there exists $\lambda$ in the extended centroid of $R$ such that $F(x)=\lambda x$ for all $x \in R$.

$(2) \Rightarrow(3)$ Suppose that

$$
F\left(\left[x, x^{*}\right]\right)=\left[F(x), x^{*}\right]-d\left(x^{*}\right) \circ x \text { for all } x \in R .
$$

Replacing $x$ by $x+y$, we obtain

$$
F\left(\left[x, y^{*}\right]\right)+F\left(\left[y, x^{*}\right]\right)=\left[F(x), y^{*}\right]+\left[F(y), x^{*}\right]-d\left(x^{*}\right) \circ y-d\left(y^{*}\right) \circ x
$$

thereby obtaining

$$
F([x, y])+F\left(\left[y^{*}, x^{*}\right]\right)=[F(x), y]+\left[F\left(y^{*}\right), x^{*}\right]-d\left(x^{*}\right) \circ y^{*}-d(y) \circ x .
$$

Replacing $y$ by $y h$ in (7), where $h \in Z(R) \cap H(R) \backslash\{0\}$, it is obvious to see that

$$
x y d(h)=0 \text { for all } x, y \in R .
$$

In light of primeness, it follows that $d(h)=0$ for all $h \in Z(R) \cap H(R)$. Substituting $y s$ for $y$ in (7), with $0 \neq s \in Z(R) \cap S(R)$, one can obtain

$$
F([x, y])-F\left(\left[y^{*}, x^{*}\right]\right)=[F(x), y]-\left[F\left(y^{*}\right), x^{*}\right]+d\left(x^{*}\right) \circ y^{*}-d(y) \circ x .
$$

Comparing (7) with (8), we find that

$$
F([x, y])=[F(x), y]-d(y) \circ x \text { for all } x, y \in R \text {. }
$$

Another use of Lemma 2.3, gives the required result. 
In [3, Theorem 2.8] it is proved that if $R$ is a 2-torsion free semi-prime ring with a generalized derivation $F$ associated with a nonzero derivation $d$ such that

$$
F([x, y])=[F(x), y]+[d(y), x] \text { for all } \in I(\diamond)
$$

where $I$ is a nonzero ideal of $R$, then $R$ contains a nonzero central ideal.

Our aim in the following theorem is to study the case the identity $(\diamond)$ is replaced by a more general algebraic identity. More precisely, we classify the generalized derivation.

Theorem 2.5. Let $(R, *)$ be a 2-torsion free prime ring with involution of the second kind and $F$ a generalized derivation associated with a derivation $d$ such that $F\left(\left[x, x^{*}\right]\right)=\left[F(x), x^{*}\right]+\left[d\left(x^{*}\right), x\right]$ for all $x \in R$. Then either $R$ is commutative or there exists $\lambda$ in the extended centroid of $R$ such that $F(x)=\lambda x$ for all $x \in R$.

Proof. Assume that

$$
F\left(\left[x, x^{*}\right]\right)=\left[F(x), x^{*}\right]+\left[d\left(x^{*}\right), x\right] \text { for all } x \in R .
$$

By linearization we get

$$
F\left(\left[x, y^{*}\right]\right)+F\left(\left[y, x^{*}\right]\right)=\left[F(x), y^{*}\right]+\left[F(y), x^{*}\right]+\left[d\left(x^{*}\right), y\right]+\left[d\left(y^{*}\right), x\right]
$$

which leads to

$$
F([x, y])+F\left(\left[y^{*}, x^{*}\right]\right)=[F(x), y]+\left[F\left(y^{*}\right), x^{*}\right]+\left[d\left(x^{*}\right), y^{*}\right]+[d(y), x] .
$$

Taking $y=y h$ in (11), where $h \in Z(R) \cap H(R) \backslash\{0\}$, we obtain

$$
[x, y] d(h)=0 \text { for all } x, y \in R .
$$

Since $R$ is prime, it follows that either $d(h)=0$ or $R$ is commutative.

Assume that $d(h)=0$ for all $h \in Z(R) \cap H(R)$; writing ys instead of $y$ in (11) with $s \in Z(R) \cap S(R) \backslash\{0\}$, we find that

$$
F([x, y])-F\left(\left[y^{*}, x^{*}\right]\right)=[F(x), y]-\left[F\left(y^{*}\right), x^{*}\right]-\left[d\left(x^{*}\right), y^{*}\right]+[d(y), x]
$$

Using (11) together with (12), we find that

$$
F([x, y])=[F(x), y]+[d(y), x] \text { for all } x, y \in R .
$$

In view of Lemma 2.3 , there exists $\lambda$ in the extended centroid of $R$ such that $F(x)=\lambda x$ for all $x \in R$. 
In [10, Theorems 2.3 and 2.4] it is proved that if $R$ is a 2-torsion free semiprime ring admitting a generalized derivation $F$ with associated nonzero derivation $d$ satisfying any one of the following conditions

$$
\text { i) } F([x, y])=F(x) \circ y-d(y) \circ x \quad \text { ii) } F(x \circ y)=[F(x), y]+[d(y), x]
$$

for all $x, y$ in a nonzero ideal $I$ of $R$, then $R$ contains a nonzero central ideal.

Our next purpose in the following theorem is to study generalized derivations $(F, d)$ satisfying the above identities in the case of prime rings with involution. We have studied this problem and proved that such conditions cannot be considered as commutativity criteria. Moreover, we successfully provide a complete description of those generalized derivations.

Theorem 2.6. Let $(R, *)$ be a 2-torsion free prime ring with involution of the second kind. If $R$ admits a generalized derivation $F$ associated with a derivation d, then the following assertions are equivalent:

(1) $F\left(\left[x, x^{*}\right]\right)=F(x) \circ x^{*}-d\left(x^{*}\right) \circ x$ for all $x \in R$;

(2) $F\left(x \circ x^{*}\right)=\left[F(x), x^{*}\right]+\left[d\left(x^{*}\right), x\right]$ for all $x \in R$;

(3) $F=0$.

Proof. (1) $\Rightarrow(3)$ By the assumption, we have

$$
F\left(\left[x, x^{*}\right]\right)=F(x) \circ x^{*}-d\left(x^{*}\right) \circ x \text { for all } x \in R .
$$

A linearization of (14) yields

$F\left(\left[x, y^{*}\right]\right)+F\left(\left[y, x^{*}\right]\right)=F(x) \circ y^{*}+F(y) \circ x^{*}-d\left(x^{*}\right) \circ y-d\left(y^{*}\right) \circ x$ for all $x, y \in R$, and thus

$$
F([x, y])+F\left(\left[y^{*}, x^{*}\right]\right)=F(x) \circ y+F\left(y^{*}\right) \circ x^{*}-d\left(x^{*}\right) \circ y^{*}-d(y) \circ x .
$$

Replacing $y$ by $y h$ in (15), where $h \in Z(R) \cap H(R) \backslash\{0\}$, one can easily verify that

$$
\left(x y-x^{*} y^{*}\right) d(h)=0 \text { for all } x, y \in R .
$$

In view of primeness, the above expression yields that either $x y-x^{*} y^{*}=0$ or $d(h)=0$.

Suppose that

$$
x y-x^{*} y^{*}=0 \text { for all } x, y \in R .
$$

If we put $y=h$, where $h \in Z(R) \cap H(R) \backslash\{0\}$, we get $x-x^{*}=0$.

Replacing $y$ by $s$, where $s \in Z(R) \cap S(R) \backslash\{0\}$, we obtain $x+x^{*}=0$. In such a way 
that $R=\{0\}$, a contradiction. Accordingly, $d(h)=0$ for all $h \in Z(R) \cap H(R)$.

Replace $y$ by $y s$ in (15), where $s \in Z(R) \cap S(R) \backslash\{0\}$, we have

$$
F([x, y])-F\left(\left[y^{*}, x^{*}\right]\right)=F(x) \circ y-F\left(y^{*}\right) \circ x^{*}+d\left(x^{*}\right) \circ y^{*}-d(y) \circ x
$$

Comparing (15) with (18), one has

$$
F([x, y])=F(x) \circ y-d(y) \circ x \text { for all } x, y \in R .
$$

In particular, $y=h$ in (19), implies that $F=0$.

$(2) \Rightarrow(3)$ We are assuming that

$$
F\left(x \circ x^{*}\right)=\left[F(x), x^{*}\right]+\left[d\left(x^{*}\right), x\right] \text { for all } x \in R .
$$

Linearizing the above relation, one can see that

$$
F\left(x \circ y^{*}\right)+F\left(y \circ x^{*}\right)=\left[F(x), y^{*}\right]+\left[F(y), x^{*}\right]+\left[d\left(x^{*}\right), y\right]+\left[d\left(y^{*}\right), x\right]
$$

and therefore

$$
F(x \circ y)+F\left(y^{*} \circ x^{*}\right)=[F(x), y]+\left[F\left(y^{*}\right), x^{*}\right]+\left[d\left(x^{*}\right), y^{*}\right]+[d(y), x] .
$$

Replacing $y$ by $y h$ in (21), where $h \in Z(R) \cap H(R) \backslash\{0\}$, we obtain

$$
\left(x \circ y+y^{*} \circ x^{*}\right) d(h)=\left(\left[y^{*}, x^{*}\right]+[y, x]\right) d(h) \text { for all } x, y \in R
$$

then

$$
\left(x y+x^{*} y^{*}\right) d(h)=0 \text { for all } x, y \in R .
$$

Arguing as above, equation (23) implies that

$$
d(h)=0 \text { for all } h \in Z(R) \cap H(R) .
$$

Replace $y$ by $y s$ in (21), where $s \in Z(R) \cap S(R) \backslash\{0\}$, we obtain

$$
F(x \circ y)-F\left(y^{*} \circ x^{*}\right)=[F(x), y]-\left[F\left(y^{*}\right), x^{*}\right]-\left[d\left(x^{*}\right), y^{*}\right]+[d(y), x] .
$$

Adding relations (21) and (24), we get

$$
F(x \circ y)=[F(x), y]+[d(y), x] \text { for all } x, y \in R .
$$

Replacing $y$ by $h$ in (25), we find that

$$
F(x) h=0 \text { for all } x \in R .
$$

Then we conclude that $F=0$.

As an application of our result, the following theorem constitute a suitable version of [10, Theorems 2.3 and 2.4] for the class of prime rings with involution. 
Theorem 2.7. Let $(R, *)$ be a 2-torsion free prime ring with involution of the second kind. If $R$ admits a generalized derivation $F$ associated with a derivation d, then the following assertions are equivalent:

(1) $F([x, y])=F(x) \circ y-d(y) \circ x$ for all $x, y \in R$;

(2) $F(x \circ y)=[F(x), y]+[d(y), x]$ for all $x, y \in R$;

(3) $F=0$.

The following theorem provides some commutativity criteria for prime rings with involution involving generalized derivations. Furthermore, we classify such generalized derivations.

Theorem 2.8. Let $(R, *)$ be a 2-torsion free prime ring with involution of the second kind. If $R$ admits a nonzero generalized derivation $F$ associated with a derivation d satisfying one of the following conditions :

(1) $F\left(\left[x, x^{*}\right]\right)=\left[d(x), x^{*}\right]+d\left(x^{*}\right) \circ x$ for all $x \in R$,

(2) $F\left(\left[x, x^{*}\right]\right)=\left[d(x), x^{*}\right]-d\left(x^{*}\right) \circ x$ for all $x \in R$,

(3) $F\left(\left[x, x^{*}\right]\right)=d(x) \circ x^{*}-d\left(x^{*}\right) \circ x$ for all $x \in R$,

then $R$ is commutative. Furthermore, there exists $\lambda$ in the extended centroid of $R$ such that $F(x)=\lambda x$ for all $x \in R$.

Proof. (1) Suppose that

$$
F\left(\left[x, x^{*}\right]\right)=\left[d(x), x^{*}\right]+d\left(x^{*}\right) \circ x \text { for all } x \in R .
$$

Linearizing (26), one can see that

$$
F([x, y])+F\left(\left[y^{*}, x^{*}\right]\right)=[d(x), y]+\left[d\left(y^{*}\right), x^{*}\right]+d\left(x^{*}\right) \circ y^{*}+d(y) \circ x .
$$

Replacing $y$ by $y h$ in (27), where $h \in Z(R) \cap H(R) \backslash\{0\}$, we obtain

$$
[x, y] d(h)=(y \circ x) d(h) \text { for all } x, y \in R,
$$

thereby obtaining

$$
y x d(h)=0 \text { for all } x, y \in R .
$$

In light of primeness, the above expression assures that $d(h)=0$.

Substituting $y s$ for $y$ in (27), where $s \in Z(R) \cap S(R) \backslash\{0\}$, we obtain

$$
F([x, y])-F\left(\left[y^{*}, x^{*}\right]\right)=[d(x), y]-\left[d\left(y^{*}\right), x^{*}\right]-d\left(x^{*}\right) \circ y^{*}+d(y) \circ x
$$

Comparing (27) with (30), one can verify that

$$
F([x, y])=[d(x), y]+d(y) \circ x \text { for all } x, y \in R .
$$


Replacing $x$ by $h$ in (31), where $h \in Z(R) \cap S(R) \backslash\{0\}$, we obtain

$$
d(y) h=0 \text { for all } y \in R,
$$

which proves that $d=0$. Then our hypothesis reduces to $F\left(\left[x, x^{*}\right]\right)=0$ for all $x \in R$. Since $F \neq 0$, then $R$ is commutative by ([8], Theorem 1 ).

Accordingly, equation (31) becomes

$$
F(x) y-F(y) x=0 \text { for all } x, y \in R .
$$

Replacing $y$ by $y z$, we find that

$$
F(x) z y-x z F(y)=0 \text { for all } x, y, z \in R .
$$

By view of Lemma 2.2, there exists $\lambda$ in the extended centroid of $R$ such that $F(x)=\lambda x$ for all $x \in R$.

(2) Suppose that

$$
F\left(\left[x, x^{*}\right]\right)=\left[d(x), x^{*}\right]-d\left(x^{*}\right) \circ x \text { for all } x \in R .
$$

A linearization of (34) leads to

$$
F([x, y])+F\left(\left[y^{*}, x^{*}\right]\right)=[d(x), y]+\left[d\left(y^{*}\right), x^{*}\right]-d\left(x^{*}\right) \circ y^{*}-d(y) \circ x .
$$

Replacing $y$ by $y h$ in (35), where $h \in Z(R) \cap H(R) \backslash\{0\}$, we obtain

$$
([x, y]+y \circ x) d(h)=0 \text { for all } x, y \in R
$$

in such a way that

$$
x y d(h)=0 \text { for all } x, y \in R
$$

proving that $d(h)=0$ for all $h \in Z(R) \cap H(R)$.

Replacing $y$ by $y s$ in (35), where $0 \neq s \in Z(R) \cap S(R)$, we arrive at

$$
F([x, y])-F\left(\left[y^{*}, x^{*}\right]\right)=[d(x), y]-\left[d\left(y^{*}\right), x^{*}\right]+d\left(x^{*}\right) \circ y^{*}-d(y) \circ x
$$

Comparing (35) with (38), it follows that

$$
F([x, y])=[d(x), y]-d(y) \circ x \text { for all } x, y \in R .
$$

Writing $y x$ instead of $y$ in (39) and invoking (39), we obtain $x y d(x)=0$ so that $d=0$. Therefore our identity reduces to $F\left[x, x^{*}\right]=0$ for all $x \in R$. Using the same techniques as used above we conclude that $R$ is commutative and $d=0$. Finally, there exists $\lambda$ in the extended centroid of $R$ such that $F(x)=\lambda x$ for all $x \in R$.

(3) Assume that

$$
F\left(\left[x, x^{*}\right]\right)=d(x) \circ x^{*}-d\left(x^{*}\right) \circ x \text { for all } x \in R .
$$


A linearization of (40) implies that

$$
F([x, y])+F\left(\left[y^{*}, x^{*}\right]\right)=d(x) \circ y+d\left(y^{*}\right) \circ x^{*}-d\left(x^{*}\right) \circ y^{*}-d(y) \circ x .
$$

Replacing $y$ by $y h$ in (41), where $h \in Z(R) \cap H(R) \backslash\{0\}$, it is obvious to verify that

$$
\left(x y-x^{*} y^{*}\right) d(h)=0 \text { for all } x, y \in R .
$$

Since equation (42) is the same as equation (16), arguing as above, we are forced to conclude $d(h)=0$.

Replacing $y$ by ys in (41), where $s$ is a nonzero element in $Z(R) \cap S(R)$, we have

$$
F([x, y])-F\left(\left[y^{*}, x^{*}\right]\right)=d(x) \circ y-d\left(y^{*}\right) \circ x^{*}+d\left(x^{*}\right) \circ y^{*}-d(y) \circ x
$$

Combining (41) with (43), one has

$$
F([x, y])=d(x) \circ y-d(y) \circ x \text { for all } x, y \in R .
$$

Writing $h$ instead of $y$ in (44), it follows that

$$
d(x) h=0 \text { for all } x \in R .
$$

which proves that $d=0$, and (40) becomes $F\left(\left[x, x^{*}\right]\right)=0$ for all $x \in R$. Consequently, $d=0, R$ is commutative and there exists $\lambda$ in the extended centroid of $R$ such that $F(x)=\lambda x$ for all $x \in R$.

As an application of Theorem 2.8, we have the following result.

Theorem 2.9. Let $(R, *)$ be a 2-torsion free prime ring with involution of the second kind. If $R$ admits a generalized derivation $F$ associated with a derivation d satisfying one of the following conditions:

(1) $F([x, y])=[d(x), y]+d(y) \circ x$ for all $x, y \in R$,

(2) $F([x, y])=[d(x), y]-d(y) \circ x$ for all $x, y \in R$,

(3) $F([x, y])=d(x) \circ y-d(y) \circ x$ for all $x, y \in R$,

then $R$ is commutative. Furthermore, there exists $\lambda$ in the extended centroid of $R$ such that $F(x)=\lambda x$ for all $x \in R$.

The following example proves that the primeness hypothesis in Theorem 2.8 is not superfluous.

Example 2.10. Let us consider $R=M_{2}(\mathbb{Z})$ and define $\left(\begin{array}{ll}a & b \\ c & d\end{array}\right)^{*}=\left(\begin{array}{cc}d & -b \\ -c & a\end{array}\right)$ and $F\left(\begin{array}{cc}a & b \\ c & d\end{array}\right)=\left(\begin{array}{cc}a & b \\ 0 & 0\end{array}\right)$. Then $F$ is a left multiplier and $(R, *)$ is a prime ring with involution of the first kind such that $\left[x, x^{*}\right]=0 \forall x \in R$. 
Set $\mathcal{R}=R \times \mathbb{C}$, then it is obvious to verify that $(\mathcal{R}, \sigma)$ is a semi-prime ring with involution of the second kind where $\sigma(r, z)=\left(r^{*}, \bar{z}\right)$.

Moreover, if we put

$$
\mathcal{F}(r, z)=(F(r), 0)
$$

then $\mathcal{F}$ is a left multiplier satisfying the conditions of Theorems 2.8 but $\mathcal{R}$ is not commutative.

The following example proves that the condition $*$ is of the second kind is necessary in Theorem 2.8 .

Example 2.11. Let us consider $R=\left\{\left(\begin{array}{ll}a & b \\ c & d\end{array}\right) \mid a, b, c, d \in \mathbb{Z}\right\}$ and $\left(\begin{array}{ll}a & b \\ c & d\end{array}\right)^{*}=$ $\left(\begin{array}{cc}d & -b \\ -c & a\end{array}\right)$. It is straightforward to check that $(R, *)$ is a prime ring with involution of the first kind such that

$$
\left[x, x^{*}\right]=0 \text { for all } x \in R .
$$

Furthermore, the mapping $F: R \longrightarrow R$ defined by

$$
F\left(\begin{array}{ll}
a & b \\
c & d
\end{array}\right)=\left(\begin{array}{ll}
a & b \\
0 & 0
\end{array}\right)
$$

is a left multiplier that satisfies conditions of Theorem 2.8 however $R$ is not commutative.

Acknowledgement. The authors would like to thank the referee for the valuable suggestions and comments.

\section{References}

[1] S. Ali and N. A. Dar, On *-centralizing mapping in rings with involution, Georgian Math. J., 21(1) (2014), 25-28.

[2] S. Ali, N. A. Dar and M. Asci, On derivations and commutativity of prime rings with involution, Georgian Math. J., 23(1) (2016), 9-14.

[3] M. Ashraf, N. Rehman, S. Ali and M. R. Mozumder, On semiprime rings with generalized derivations, Bol. Soc. Parana. Mat., (3), 28(2) (2010), 25-32.

[4] M. Brešar, Semiderivations of prime rings, Proc. Amer. Math. Soc., 108(4) (1990), 859-860.

[5] M. Brešar, On generalized biderivations and related maps, J. Algebra, 172(3) (1995), 764-786. 
[6] V. De Filippis, N. Rehman and A. Ansari, Lie ideals and generalized derivations in semiprime rings, Iran. J. Math. Sci. Inform., 10(2) (2015), 45-54.

[7] B. Hvala, Generalized derivations in rings, Comm. Algebra, 26 (1998), 11471166.

[8] M. A. Idrissi and L. Oukhtite, Some commutativity theorems for rings with involution involving generalized derivations, Asian-Eur. J. Math., 12(1) (2019), 1950001 (11 pp).

[9] C. Lanski, Differential identities, Lie ideals and Posner's theorems, Pacific J. Math., 134(2) (1988), 275-297.

[10] M. R. Khan and M. M. Hasnain, On semiprime rings with generalized derivations, Kyungpook Math. J., 53(4) (2013), 565-571.

[11] T. K. Lee, Generalized derivations of left faithful rings, Comm. Algebra, 27 (1999), 4057-4073.

[12] B. Nejjar, A. Kacha, A. Mamouni and L. Oukhtite, Certain commutativity criteria for rings with involution involving generalized derivations, Georgian Math. J., 27(1) (2020), 133-139.

[13] L. Oukhtite and A. Mamouni, Generalized derivations centralizing on Jordan ideals of rings with involution, Turkish J. Math., 38(2) (2014), 225-232.

[14] E. C. Posner, Derivations in prime rings, Proc. Amer. Math. Soc., 8 (1957), 1093-1100.

M. A. Idrissi (Corresponding Author)

Department of Mathematics and Informatics

Polydisciplinary Faculty

Sultan Moulay Slimane University

Beni Mellal, Box 592, Morocco

e-mail: myabdallahidrissi@gmail.com

L. Oukhtite

Department of Mathematics

Faculty of Science and Technology

University S. M. Ben Abdellah

Fez, Box 2202, Morocco

e-mail: oukhtitel@hotmail.com 